\title{
The enigmatic ERH protein: its role in cell cycle, RNA splicing and cancer
}

\author{
Meng-Tzu Weng, Ji Luo \\ Medical Oncology Branch, Center for Cancer Research, National Cancer Institute, Bethesda, MD 20892, USA \\ $\triangle$ Correspondence: ji.luo@nih.gov \\ Received July 11, 2013 Accepted August 26, 2013
}

\begin{abstract}
Enhancer of rudimentary homolog (ERH) is a small, highly conserved protein among eukaryotes. Since its discovery nearly $\mathbf{2 0}$ years ago, its molecular function has remained enigmatic. It has been implicated to play a role in transcriptional regulation and in cell cycle. We recently showed that ERH binds to the Sm complex and is required for the mRNA splicing of the mitotic motor protein CENP-E. Furthermore, cancer cells driven by mutations in the KRAS oncogene are particularly sensitive to RNAi-mediated suppression of ERH function, and ERH expression is inversely correlated with survival in colorectal cancer patients whose tumors harbor KRAS mutation. These recent findings indicate that ERH plays an important role in cell cycle through its mRNA splicing activity and is critically required for genomic stability and cancer cell survival.
\end{abstract}

KEYWORDS enhancer of rudimentary homolog, mRNA splicing, mitosis, CENP-E, KRAS, colorectal cancer

\section{DISCOVERY AND EVOLUTIONARY CONSERVATION OF THE ERH PROTEIN}

The first $E R H$ ortholog was discovered by the Tsubota lab from an insertional mutagenesis screen in Drosophila for mutants that enhanced the wing phenotype of a hypomorphic allele of the rudimentary gene (Wojcik et al., 1994). A mutant allele of the enhancer of rudimentary $[e(r)]$ gene resulting from $\mathrm{P}$ element insertion led to a more severe wing truncation in the mutant rudimentary background. As the rudimentary gene encodes a CAD-like enzyme in the pyrimidine biosynthetic pathway, it was suggested that $e(r)$ might play a role in the regulation of pyrimidine metabolism. Flies with hypomorphic mutations in $e(r)$ are viable, but homozygous deletion of this gene severely reduces viability (Roykhman et al., 2007). Thus $e(r)$ is likely to encode a critical, if not essential protein in Drosophila.
Since its discovery, orthologs of the $E R H$ gene have been identified in most eukaryotes and they encode a small protein that is highly conserved throughout metazoans and plants (Weng et al., 2012) (Fig. 1). ERH orthologs can be traced back to fission yeasts including Schizosaccharomyces pombe ( $S$. pombe) and Schizosaccharomyces japonicus, but not the budding yeast Saccharomyces cerevisiae (Krzyzanowski et al., 2012; Weng et al., 2012). Amino acid sequence similarity is a striking $\sim 80 \%$ between human and Dyctyostelium and that between Human and Arabidopsis is $\sim 78 \%$. Such high degree of sequence conservation strongly implicates conserved molecular function of ERH across eukaryotes.

\section{STRUCTURE AND REGULATION OF ERH}

The human ERH gene is located on chromosome 14q24.1 and is ubiquitously expressed in all tissues (Smyk et al., 2006). EGFP-tagged ERH primarily localizes to the nucleus but is excluded from the nucleolus. During mitosis, ERH is distributed throughout the cell but excluded from chromatin (Smyk et al., 2006; Lukasik et al., 2008; Weng et al., 2012). These findings indicate a nuclear function of ERH. Mammalian ERH protein adopts a RAGNYA type $\alpha+\beta$ fold consisting of a fourstranded antiparallel beta sheet packed adjacent to three amphipathic $\alpha$-helices (Arai et al., 2005; Li et al., 2005; Wan et al., 2005; Balaji and Aravind, 2007; Jin et al., 2007) (Fig. 1). In solution, purified ERH protein forms homodimers, suggesting ERH might function as a dimer in cell. The Drosophila E(r) protein can be phosphorylated by casein kinase II (CKII) in vitro (Gelsthorpe et al., 1997), and mutations in $\mathrm{E}(\mathrm{r})$ at two CKII consensus sites T18 and S24 compromise $\mathrm{E}(\mathrm{r})$ function in vivo (Gelsthorpe et al., 2006). Although it remains to be determined whether CKII also phosphorylates ERH in mammalian cells, mass-spectrometry data indicate that S24 might be a major phosphorylation site on human ERH. Furthermore, K41 and K90 of human ERH appear to be two major sites for ubiquitination (http://www.phosphosite.org). Thus the function of ERH 


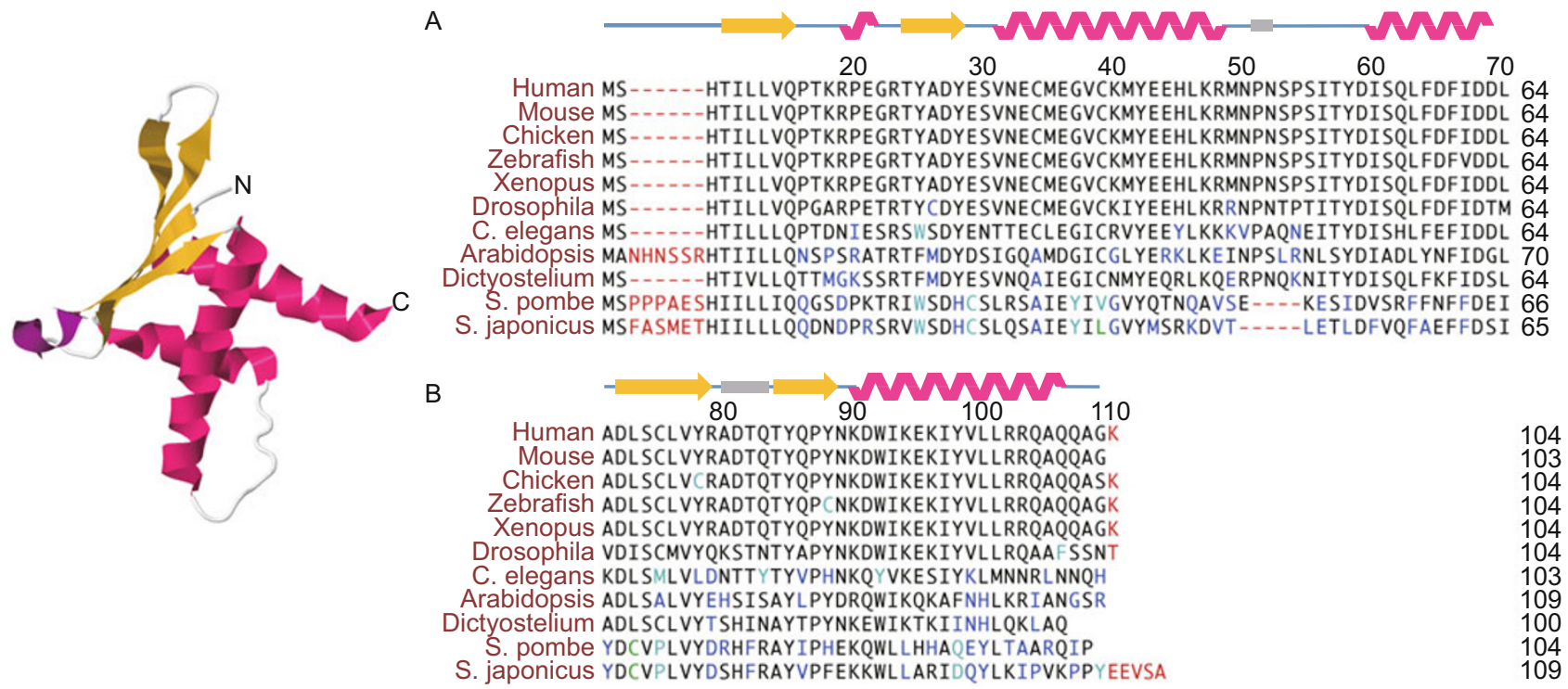

Figure 1. Crystal structure of human ERH protein and sequence homology among ERH orthologs. (A) Crystal structure of human ERH protein. Image was generated with the human ERH crystal structure 2NML (Jin et al., 2007) using the PDB viewer (http://www.rcsb. org/pdb/). (B) Sequence homology among ERH orthologs. Secondary structure of $\alpha$-helix and $\beta$-strand corresponding to the human ERH sequence in the crystal structure are indicated on top.

could be subject to regulation by both phosphorylation and ubiquitination.

\section{THE ROLE OF ERH IN TRANSCRIPTION REGULATION}

Genetic evidence from Drosophila indicates that $E(r)$ might function as a transcriptional regulator, although it does not seem to strongly affect rudimentary transcript levels in adult flies (Wojcik et al., 1994). The Xenopus ERH protein, XERH, was identified in a yeast two-hybrid screen to interact with the transcriptional co-activator dimerization cofactor of HNF1/ pterin-4a-carbinolamine dehydratase $(\mathrm{DCoH} / \mathrm{PCD})$ (Pogge von Strandmann et al., 2001). DCoH is a multifunctional protein that was first identified as a positive cofactor for the HNF1 homeobox transcription factor. DCoH stabilizes HNF1a dimers and enhances its transcriptional activity by forming a stable tetrameric DCoH-HNF1a complex without changing the DNA binding characteristics of HNF1a (Mendel et al., 1991). In HeLa cells the co-expression of Xenopus DCoH and HNF1a leads to stronger activation of an HNF1-responsive reporter than either protein alone, and over-expression of XERH inhibits the activation of this reporter by DCoH and HNF1a (Pogge von Strandmann et al., 2001).

ERH might also play a role in transcriptional elongation. ERH interacts with both the RNA Pol II C-terminal domain phosphatase FCP1 and the transcription factor SPT5, which regulates RNA Pol II elongation ( Kwak et al., 2003; Amente et al., 2005). Both FCP1 and SPT5 are substrates of the methyltransferase PRMT5, which co-purifies with these proteins together with ERH. However, it is not clear whether ERH binding influences the function of FCP1 and SPT5 and whether ERH plays a role in regulating the activity of Pol II.

We have recently shown that RNAi-mediated knockdown of ERH in human colorectal cancer cell line DLD-1 results in altered expression of a small set of genes, the majority of which are down-regulated (Weng et al., 2012). Further studies are necessary to establish whether the down regulation of these genes occur at the transcriptional level. Because our study uncovered a role of ERH in mRNA splicing (see below), it is possible that the down-regulation of these ERH target genes is a consequence of mRNA splicing defects. Thus the in vivo evidence supporting a role of endogenous ERH in transcriptional regulation is relatively weak and further studies are necessary to confirm the role of ERH in this context.

\section{THE ROLE OF ERH IN CELL CYCLE AND MITOSIS}

The $E R H$ ortholog gene $e r h 1^{+}$in the fission yeast S. pombe is not essential but is required for optimal growth under stress conditions (Krzyzanowski et al., 2012). In S. pombe erh $1^{+}$is expressed in all growth stages and disruption of $\mathrm{erh}^{+}$by homologous recombination results in aberrant cell morphology and reduced growth rate. Auxotrophic erh1 $\Delta$ mutants show stronger $G_{1}$ arrest upon nitrogen starvation and are hypersensitive to agents that cause hyperosmotic stress (sorbitol), DNA damage (hydroxyurea), or membrane disruption (SDS). Interestingly, re-expression of human ERH in these mutants rescues sorbitol sensitivity but not hydroxyurea or SDS sensitivity, indicating a partial conservation of ERH function between human and yeast.

The human ERH protein has been found to interact with 
SKAR and CIZ1 in yeast-two hybrid screens (Smyk et al., 2006; Lukasik et al., 2008). SKAR is a nuclear protein that recruits the ribosomal protein S6 kinase, S6K1, to newly synthesized mRNAs to stimulate their translation (Richardson et al., 2004; Ma et al., 2008). ClZ1 is a zinc finger protein that plays a role in cell cycle and DNA replication through its interaction with the CDK inhibitor p21 cip1/waf1 and cyclins $E$ and A (Mitsui et al., 1999; Copeland et al., 2010). Although it remains to be confirmed that endogenous ERH interacts with endogenous SKAR and $\mathrm{ClZ1}$ to regulate cell growth and DNA replication, these studies suggest a role of ERH in cell cycle regulation.

Recently, we and the Kimura lab independently discovered that in mammalian cells $\mathrm{ERH}$ is required for chromosome segregation during mitosis (Fujimura et al., 2012; Weng et al., 2012). RNAi-mediated $E R H$ knockdown leads to $G_{2} / M$ arrest and chromosome congression defect. In mitosis, chromosomes must align at the metaphase mid-plate and achieve bi-oriented attachment to opposing spindle poles prior to the onset of anaphase to ensure proper segregation of sister chromatids. This process is orchestrated by a large number of proteins (Kops et al., 2010; Tanaka, 2013). During chromosome congression, individual kinetochores are first captured laterally on microtubules and moved to spindle pole by minus end-directed dynein motor proteins. There kinetochore attachment is converted to an end-on attachment with the help of the KNL1-Mis12-Ndc80 network of kinetochore proteins. Transportation of kinetochores towards the metaphase mid-plate along $\mathrm{K}$ fibers is carried out by the plus end-directed motor protein CENP-E. Proper kinetochore attachment is sensed by the spindle assembly checkpoint (SAC). SAC remains active in the presence of unattached, merotelically attached or syntelically attached kinetochores to inhibit the onset of anaphase. Only when all kinetochores are correctly attached is the SAC silenced and sister chromatids allowed to segregate.

ERH depletion results in the loss of CENP-E from kinetochore and consequently chromosomes fail to congress in a timely fashion (Fujimura et al., 2012; Weng et al., 2012). Live cell videomicroscopy in U2OS cells indicates that ERH depletion increases the duration of mitosis by approximately 6-fold due to defective chromosome congression. The loss of CENP-E from kinetochore is due to the loss of CENP-E expression, as both CENP-E protein and mRNA levels are severely reduced in the absence of ERH (see below). ERH appears to be selectively required for the expression of CENP-E but not all mitotic proteins: the expression and localization of the Ndc80 complex subunit Hec1, and the SAC proteins Bub1, BubR1, and MAD2 are not affected by ERH knockdown. Furthermore, in the absence of ERH the SAC remains intact as cells can still arrest stably in the presence of the microtubule depolymerization agent nocodazole (Fujimura et al., 2012; Weng et al., 2012).

In addition to CENPE, ERH appears to also regulate other cell cycle genes. Gene expression analysis indicates that a small set of cell cycle and DNA replication/repair genes are down regulated following $E R H$ knockdown. Among these are SMC4, KIF20, ATM, FANCD, MRE11A, RAD50, and RAD54B
(Weng et al., 2012). Together these findings suggest that ERH is required for cell cycle progression and in particular, normal chromosome segregation during mitosis.

\section{ERH IS AN RNA SPLICING FACTOR}

$\mathrm{ERH}$ is a nuclear protein localized to the nucleoplasm and excluded from the nucleolus and chromatin (Smyk et al., 2006; Lukasik et al., 2008; Weng et al., 2012). Given ERH has been implicated as a transcription factor, we initially tested whether ERH can bind to the CENPE promoter. However, chromatin immunoprecipitation (ChIP) experiments failed to detect ERH localization to the CENPE promoter, thus prompting us to examine alternative mechanisms of CENPE regulation by ERH. Using SILAC-mass spectrometry, we unexpectedly identified the Sm complex subunit SNRPD3 as a binding partner for ERH. Reciprocal IP experiments further confirmed the interaction between endogenous ERH and SNRPD3 (Weng et al., 2012). SNRPD3 is a subunit of the heteroheptameric Sm complex that binds to the spliceosome U1, U2, U4, and U5 small nuclear RNAs (snRNAs) to promote their 5'-cap hypermethylation and maturation (Beggs, 2005). Sm is in turn targeted to these snRNAs by the SMN complex (Battle et al., 2006) and a recent SMN interactome study identified ERH as an associated protein (Fuller et al., 2010). These findings indicate that $\mathrm{ERH}$ is likely to associate with core mRNA splicing factors through its binding to the Sm complex.

Functional evidence supporting a role of ERH in mRNA splicing came from the analysis of CENPE mRNA. Depletion of ERH results in a loss of mature CENPE mRNA that accounts for the loss of CENP-E protein at the kinetochore. RT-qPCR analysis of multiple CENPE exon-intron junctions indicates that CENPE pre-mRNA fails to splice efficiently in the absence of $E R H$, and unspliced/mis-spliced CENPE pre-mRNA is likely degraded through the nonsense-mediated decay mRNA quality control pathway (Weng et al., 2012). In our gene expression profile analysis, 64 genes were down-regulated 2 days after ERH depletion. Many of these down-regulated genes are involved in cell cycle and DNA replication/repair pathways (Weng et al., 2012). Why ERH depletion preferentially affects cell cycle genes remains unclear and whether ERH regulates these genes through pre-mRNA splicing is unknown and requires further investigation. Interestingly, depletion of other mRNA splicing factors including the Prp19 complex and PHF5A also leads to cell cycle arrest and mitotic defects (Song et al., 2010; Hubert et al., 2013). Thus cell cycle genes might be particularly sensitive to perturbation of the mRNA splicing machinery.

\section{ERH IN MALIGNANCY}

Relatively little is known about the role of ERH in cancer. The $E R H$ gene does not appear to be a target for focal amplification/deletion or point mutation in human cancer. However, ERH expression is elevated in breast tumors and breast cancer cell lines (Zafrakas et al., 2008). ERH has been reported to interact with the tumor suppressor protein $\mathrm{H} 19$ opposite tumor sup- 
pressor (HOTS) (Onyango and Feinberg, 2011). HOTS is a nucleolar protein whose expression is frequently lost in Wilms' tumors whereas its over-expression inhibits the proliferation of Wilm's tumor cell lines. The mechanism of tumor suppression by HOTS in Wilms' tumor, and how ERH might modulate HOTS's function is currently unknown.

RNAi knockdown experiments indicate that ERH is required for tumor cell viability in a context dependent manner. Recent data from genome-wide RNAi screen in a large panel of cancer cell lines suggest that ERH is an important survival factor in certain pancreatic, breast, and ovarian cancer cell lines (Marcotte et al., 2012). We initially identified $E R H$ as a candidate KRAS synthetic lethal gene in isogenic KRAS mutant and wild type (WT) DLD-1 colorectal cancer cell lines (Luo et al., 2009a). We further showed that in a panel of colorectal cancer cell lines, ERH depletion reduced the viability of KRAS mutant cell lines more strongly than KRASWT cell lines (Weng et al., 2012). As cells driven by the RAS oncogene experience elevated mitotic stress and genomic instability (Denko et al., 1994; Luo et al., 2009a), this is likely to render them more sensitive to ERH depletion, which would further deregulate cell cycle gene expression and exacerbate mitotic stress. Thus the hypersensitivity of KRAS mutant cells to ERH depletion could reflect the non-oncogene addiction of these cells toward genes that maintain cell cycle fidelity (Luo et al., 2009b). Clinically, $E R H$ mRNA expression is associated with shorter survival among colorectal cancer patients whose tumor harbor KRAS mutations but not among patients whose tumors are WT for KRAS. Similarly, higher ERH expression also trended with shorter survival among patients whose resected lung tumors harbored KRAS mutations, but not among those whose lung tumors were WT for KRAS or harbored EGFR mutations (Weng et al., 2012). Thus high $E R H$ expression could contribute to the malignancy of KRAS mutant tumors and low $E R H$ expression might constrain the aggressiveness of these tumors.

\section{CONCLUSION}

$\mathrm{ERH}$ is a highly conserved protein identified in metazoans and plants. It is a multifunctional protein capable of regulating transcription, cell cycle, and mRNA splicing (Fig. 2). Recent studies clearly implicate a role of ERH in mitosis and the maintenance of genomic stability. Future studies will provide additional understanding on the molecular mechanisms by which ERH regulates these biological processes. Given the success of cell cycle inhibitors in cancer treatment, understanding how ERH and the mRNA splicing machinery regulate the expression of cell cycle genes could provide new insight into targeting the cell cycle machinery for cancer therapy.

\section{ACKNOWLEDGEMENTS}

This work is supported by the Liver Disease Prevention and Treatment Research Foundation of Taiwan to M.Z.W. and by the Intramural Program of the U.S. National Cancer Institute to J.L..

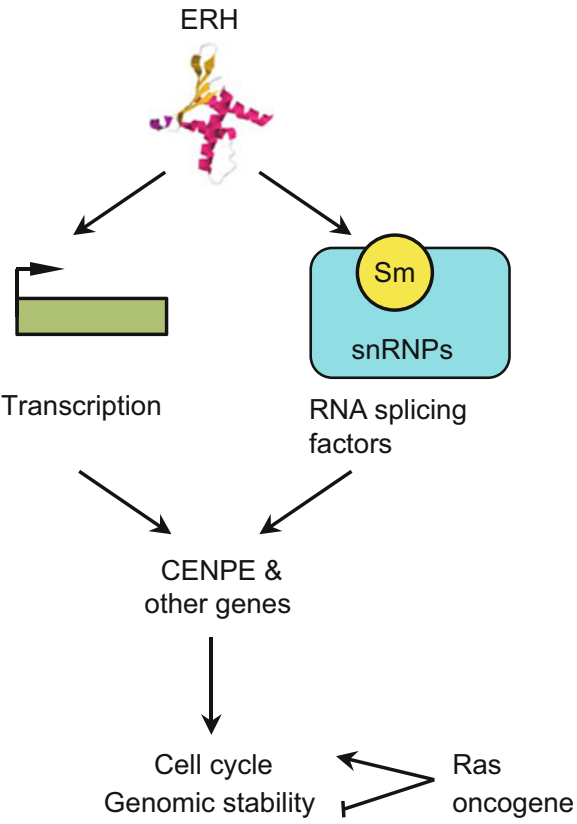

Figure 2. A model of cell cycle regulation by ERH. ERH interacts with the Sm complex and regulates the splicing of CENPE pre-mRNA. ERH also regulates the expression of additional cell cycle and DNA repair genes. Depletion of ERH synergizes with the KRAS oncogene to exacerbate cell cycle stress, this rendering KRAS mutant cancer cells particularly dependent on the function of $E R H$.

\section{ABBREVIATIONS}

ChIP, chromatin immunoprecipitation; CKII, casein kinase II; ERH, Enhancer of rudimentary homolog; HOTS, H19 opposite tumor suppressor; SAC, spindle assembly checkpoint

\section{COMPLIANCE WITH ETHICS GUIDELINES}

Meng-Tzu Weng and Ji Luo declare that they have no conflict of interest.

This article does not contain any studies with human or animal subjects performed by the any of the authors.

\section{REFERENCES}

Amente, S., Napolitano, G., Licciardo, P., Monti, M., Pucci, P., Lania, L., and Majello, B. (2005). Identification of proteins interacting with the RNAPII FCP1 phosphatase: FCP1 forms a complex with arginine methyltransferase PRMT5 and it is a substrate for PRMT5mediated methylation. FEBS Lett 579, 683-689.

Arai, R., Kukimoto-Niino, M., Uda-Tochio, H., Morita, S., Uchikubo-Kamo, T., Akasaka, R., Etou, Y., Hayashizaki, Y., Kigawa, T., Terada, T., et al. (2005). Crystal structure of an enhancer of rudimentary homolog $(\mathrm{ERH})$ at 2.1 Angstroms resolution. Protein Sci 14, 18881893.

Balaji, S., and Aravind, L. (2007). The RAGNYA fold: a novel fold with multiple topological variants found in functionally diverse nucleic acid, nucleotide and peptide-binding proteins. Nucleic Acids Res 
$35,5658-5671$.

Battle, D.J., Kasim, M., Yong, J., Lotti, F., Lau, C.-K., Mouaikel, J., Zhang, Z., Han, K., Wan, L., and Dreyfuss, G. (2006). The SMN complex: an assembly machine for RNPs. Cold Spring Harb. Symp. Quant Biol 71, 313-320.

Beggs, J.D. (2005). Lsm proteins and RNA processing. Biochem Soc Trans 33, 433-438.

Copeland, N.A., Sercombe, H.E., Ainscough, J.F.X., and Coverley, D. (2010). Ciz1 cooperates with cyclin-A-CDK2 to activate mammalian DNA replication in vitro. J Cell Sci 123, 1108-1115.

Denko, N.C., Giaccia, A.J., Stringer, J.R., and Stambrook, P.J. (1994). The human Ha-ras oncogene induces genomic instability in murine fibroblasts within one cell cycle. Proc Natl Acad Sci U S A 91, 5124-5128.

Fujimura, A., Kishimoto, H., Yanagisawa, J., and Kimura, K. (2012). Enhancer of rudimentary homolog $(\mathrm{ERH})$ plays an essential role in the progression of mitosis by promoting mitotic chromosome alignment. Biochem Biophys Res Commun 423, 588-592.

Fuller, H.R., Man, N.T., Lam, L.T., Thanh, L.T., Keough, R.A., Asperger, A., Gonda, T.J., and Morris, G.E. (2010). The SMN interactome includes Myb-binding protein 1a. J Proteome Res 9, 556-563.

Gelsthorpe, M., Pulumati, M., McCallum, C., Dang-Vu, K., and Tsubota, S.I. (1997). The putative cell cycle gene, enhancer of rudimentary, encodes a highly conserved protein found in plants and animals. Gene 186, 189-195.

Gelsthorpe, M.E., Tan, Z., Phillips, A., Eissenberg, J.C., Miller, A., Wallace, J., and Tsubota, S.I. (2006). Regulation of the Drosophila melanogaster protein, enhancer of rudimentary, by casein kinase II. Genetics 174, 265-270.

Hubert, C.G., Bradley, R.K., Ding, Y., Toledo, C.M., Herman, J., SkuttKakaria, K., Girard, E.J., Davison, J., Berndt, J., Corrin, P., et al. (2013). Genome-wide RNAi screens in human brain tumor isolates reveal a novel viability requirement for PHF5A. Genes Dev 27, 1032-1045.

Jin, T., Guo, F., Serebriiskii, I.G., Howard, A., and Zhang, Y.-Z. (2007). A 1.55 A resolution $X$-ray crystal structure of HEF2/ERH and insights into its transcriptional and cell-cycle interaction networks. Proteins 68, 427-437.

Kops, G.J.P.L., Saurin, A.T., and Meraldi, P. (2010). Finding the middle ground: how kinetochores power chromosome congression. Cell Mol Life Sci 67, 2145-2161.

Krzyzanowski, M.K.M., Kozlowska, E.E., and Kozlowski, P.P. (2012). Identification and Functional Analysis of the erh1(+) Gene Encoding Enhancer of Rudimentary Homolog from the Fission Yeast Schizosaccharomyces pombe. PLoS One 7, e49059-49059.

Kwak, Y.T., Guo, J., Prajapati, S., Park, K.-J., Surabhi, R.M., Miller, B., Gehrig, P., and Gaynor, R.B. (2003). Methylation of SPT5 regulates its interaction with RNA polymerase II and transcriptional elongation properties. Mol Cell 11, 1055-1066.

Li, H., Inoue, M., Yabuki, T., Aoki, M., Seki, E., Matsuda, T., Nunokawa, E., Motoda, Y., Kobayashi, A., Terada, T., et al. (2005). Solution structure of the mouse enhancer of rudimentary protein reveals a novel fold. J Biomol NMR 32, 329-334.

Lukasik, A., Uniewicz, K.A., Kulis, M., and Kozlowski, P. (2008). Ciz1, a p21 cip1/Waf1-interacting zinc finger protein and DNA replication factor, is a novel molecular partner for human enhancer of rudimentary homolog. FEBS J 275, 332-340.
Luo, J., Emanuele, M.J., Li, D., Creighton, C.J., Schlabach, M.R., Westbrook, T.F., Wong, K.-K., and Elledge, S.J. (2009a). A Genome-wide RNAi Screen Identifies Multiple Synthetic Lethal Interactions with the Ras Oncogene. Cell 137, 835-848.

Luo, J., Solimini, N.L., and Elledge, S.J. (2009b). Principles of cancer therapy: oncogene and non-oncogene addiction. Cell 136, 823837.

Ma, X.M., Yoon, S.-O., Richardson, C.J., Jülich, K., and Blenis, J. (2008). SKAR links pre-mRNA splicing to mTOR/S6K1-mediated enhanced translation efficiency of spliced mRNAs. Cell 133, 303313.

Marcotte, R., Brown, K.R., Suarez, F., Sayad, A., Karamboulas, K., Krzyzanowski, P.M., Sircoulomb, F., Medrano, M., Fedyshyn, Y., Koh, J.L.Y., et al. (2012). Essential gene profiles in breast, pancreatic, and ovarian cancer cells. Cancer Discov 2, 172-189.

Mendel, D.B., Khavari, P.A., Conley, P.B., Graves, M.K., Hansen, L.P., Admon, A., and Crabtree, G.R. (1991). Characterization of a cofactor that regulates dimerization of a mammalian homeodomain protein. Science 254, 1762-1767.

Mitsui, K., Matsumoto, A., Ohtsuka, S., Ohtsubo, M., and Yoshimura, A. (1999). Cloning and characterization of a novel p21(Cip1/Waf1)interacting zinc finger protein, ciz1. Biochem Biophys Res Commun 264, 457-464.

Onyango, P., and Feinberg, A.P. (2011). A nucleolar protein, H19 opposite tumor suppressor (HOTS), is a tumor growth inhibitor encoded by a human imprinted $\mathrm{H} 19$ antisense transcript. Proc Natl Acad Sci U S A 108, 16759-16764.

Pogge von Strandmann, E., Senkel, S., and Ryffel, G.U. (2001). ERH (enhancer of rudimentary homologue), a conserved factor identical between frog and human, is a transcriptional repressor. Biol Chem 382, 1379-1385.

Richardson, C.J., Bröenstrup, M., Fingar, D.C., Jülich, K., Ballif, B.A., Gygi, S., and Blenis, J. (2004). SKAR is a specific target of S6 kinase 1 in cell growth control. Curr Biol 14, 1540-1549.

Roykhman, M., Ibach, S., Harding, D., Wolff, B., Vowels, M., Gelsthorpe, M.E., Wojcik, E.J., Rizzo, N.P., and Tsubota, S.I. (2007). The generation and analysis of deficiencies within a small genomic region on the $\mathrm{X}$ chromosome of Drosophila melanogaster containing two genes, enhancer of rudimentary and CG15352. Fly (Austin) 1, 245-250.

Smyk, A., Szuminska, M., Uniewicz, K.A., Graves, L.M., and Kozlowski, P. (2006). Human enhancer of rudimentary is a molecular partner of PDIP46/SKAR, a protein interacting with DNA polymerase delta and S6K1 and regulating cell growth. FEBS J 273, 4728-4741.

Song, E.J., Werner, S.L., Neubauer, J., Stegmeier, F., Aspden, J., Rio, D., Harper, J.W., Elledge, S.J., Kirschner, M.W., and Rape, M. (2010). The Prp19 complex and the Usp4Sart3 deubiquitinating enzyme control reversible ubiquitination at the spliceosome. Genes Dev 24, 1434-1447.

Tanaka, K. (2013). Regulatory mechanisms of kinetochore-microtubule interaction in mitosis. Cell Mol Life Sci 70, 559-579.

Wan, C., Tempel, W., Liu, Z.-J., Wang, B.-C., and Rose, R.B. (2005). Structure of the conserved transcriptional repressor enhancer of rudimentary homolog. Biochemistry 44, 5017-5023.

Weng, M.-T., Lee, J.-H., Wei, S.-C., Li, Q., Shahamatdar, S., Hsu, D., Schetter, A.J., Swatkoski, S., Mannan, P., Garfield, S., et al. (2012). 
Evolutionarily conserved protein ERH controls CENP-E mRNA splicing and is required for the survival of KRAS mutant cancer cells. Proc Natl Acad Sci U S A 109, E3659-E3667.

Wojcik, E., Murphy, A.M., Fares, H., Dang-Vu, K., and Tsubota, S.I. (1994). Enhancer of rudimentaryp1, e(r)p1, a highly conserved en- hancer of the rudimentary gene. Genetics 138, 1163-1170.

Zafrakas, M., Losen, I., Knüchel, R., and Dahl, E. (2008). Enhancer of the rudimentary gene homologue $(E R H)$ expression pattern in sporadic human breast cancer and normal breast tissue. BMC Cancer $8,145$. 